\title{
Positive solutions of nonlocal boundary value problems involving integral conditions
}

\author{
Jeff R.L. WEBB \\ Department of Mathematics \\ University of Glasgow \\ Glasgow G12 8QW, UK \\ e-mail: J.Webb@maths.gla.ac.uk \\ Gennaro INFANTE \\ Dipartimento di Matematica \\ Università della Calabria \\ 87036 Arcavacata di Rende, Cosenza, Italy \\ e-mail: g.infante@unical.it
}

\begin{abstract}
We establish the existence of multiple positive solutions of nonlinear equations of the form

$$
-u^{\prime \prime}(t)=g(t) f(t, u(t)), t \in(0,1),
$$
\end{abstract}

where $g, f$ are non-negative functions, subject to various nonlocal boundary conditions. The common feature is that each can be written as an integral equation, in the space $C[0,1]$, of the form

$$
u(t)=\gamma(t) \alpha[u]+\int_{0}^{1} k(t, s) g(s) f(s, u(s)) d s
$$

where $\alpha[u]$ is a linear functional given by a Stieltjes integral but is not assumed to be positive for all positive $u$. Our new results cover many nonlocal boundary conditions previously studied on a case by case basis for particular positive functionals only, for example, many $m$-point BVPs are special cases. Even for positive functionals our methods give improvements on previous work. Also we allow weaker assumptions on the nonlinear term than were previously imposed.

2000 Mathematics Subject Classification: Primary 34B18, secondary 34B10, 47H10, $47 \mathrm{H} 30$.

Key words: Nonlocal boundary conditions, fixed point index, positive solution. 


\section{Introduction}

We establish the existence of multiple positive solutions of nonlinear nonlocal boundary value problems (BVPs) for equations of the form

$$
-u^{\prime \prime}(t)=g(t) f(t, u(t)), t \in(0,1)
$$

where $g, f$ are non-negative functions, typically $f$ is continuous and $g \in L^{1}$ may have singularities, subject to various nonlocal boundary conditions (BCs) including the following

$$
\begin{aligned}
& u(0)=0, u(1)=\alpha[u], \\
& u^{\prime}(0)=0, u(1)=\alpha[u], \\
& u(0)=0, u^{\prime}(1)=\alpha[u] .
\end{aligned}
$$

We shall take $\alpha[u]$ to be a linear functional on $C[0,1]$ given by

$$
\alpha[u]=\int_{0}^{1} u(s) d A(s)
$$

involving a Stieltjes integral with a signed measure, that is, $A$ has bounded variation. This is completely new and includes the special cases of so called $m$-point problems when $\alpha[u]=\sum_{i=1}^{m-2} \alpha_{i} u\left(\eta_{i}\right)$. These have been extensively studied by Gupta and co-authors, for example [3, 4], but they did not seek positive solutions. Existence of positive solutions for the $m$-point problem corresponding to (1.2) has been studied by Ma [14] and Zhang and Sun [22] when all the $\alpha_{i}$ are positive. The BCs (1.2) when $\alpha[u]=\int_{0}^{1} \alpha(s) u(s) d s$, with $\alpha(s) \geq 0$ continuous has been studied by Ma [15] while Karakostas and Tsamatos, [7] study the BCs (1.2) (and other more complicated BCs in [8]) with $\alpha[u]$ given by a Riemann-Stieltjes integral (with a positive measure) and include the case covered by [15]. We treat all of the above BCs (and others) in a unified way and obtain new results by allowing signed measures, but even for (positive) measures we obtain distinct improvements on $[7,15]$. In particular, we do not impose a condition which is used in both [7] and $[15]$ to reduce to the known case of a 3-point BVP. Also, when specialized to $m$-point BVPs, we obtain the existence of positive solutions when coefficients of either sign are allowed, which has not been done previously. Our conditions are essentially optimal, typically we find strict inequalities that are sufficient while non-strict inequalities are necessary.

The methodology used to treat such problems has been to write the BVP as a Hammerstein integral equation

$$
u(t)=S u(t):=\int_{0}^{1} k_{S}(t, s) g(s) f(s, u(s)) d s,
$$


for a non-negative kernel $k_{S}$ and find a solution as a fixed point of the operator $S$ by means of Krasnosel'skii's theorem or, more generally, by using the fixed point index theory of compact mappings.

We also use fixed point index theory on a suitable cone $K$ in $C[0,1]$ but our new approach is to use a new cone and to write the equation in the new form

$$
u(t)=\gamma(t) \alpha[u]+\int_{0}^{1} k(t, s) g(s) f(s, u(s)) d s:=\gamma(t) \alpha[u]+F u(t):=T u(t) .
$$

Here $\gamma(t)=1$ for BC (1.3), while $\gamma(t)=t$ for each of the BCs (1.2), (1.4). We do not suppose that $\alpha[u] \geq 0$ for all $u \geq 0$. Our new idea, which enables us to deal with a signed measure in the term $\alpha[u]=\int_{0}^{1} u(s) d A(s)$, is to include the requirement $\alpha[u] \geq 0$ to be satisfied by positive solutions into the definition of a cone that we use. Showing that our operators take values in the cone gives us, very easily, a sufficient condition for the $m$-point BVPs to have a positive kernel, which allows coefficients of both signs, and is close to necessary for the existence of positive solutions.

One advantage of our method is its generality, many BVPs can be handled by the same theory rather than on a case-by-case basis. Another advantage is that we deal with a simpler kernel in (1.7) than we would have to deal with using the explicit form of (1.6), which can appear very complicated, at the small cost of having to consider a sum of two operators. We relate the different ways of considering the fixed point problem using either (1.7) or (1.6), in particular we are able to utilise strong fixed point index results involving the largest real eigenvalue of $S$ to prove strong results for $T$. This leads to us obtaining the existence of multiple positive solutions under less stringent conditions on the nonlinearity than is needed when Krasnosel'skii's theorem is used.

For each of the three BVPs listed above we provide explicit examples of signed measures that satisfy the hypotheses, and we also deduce the conditions to be satisfied by the coefficients (of both sign) in each of the corresponding $m$ point problems. In the last section we indicate how our results apply when $u^{\prime \prime}$ is replaced by a more general form of second order operator.

\section{Preliminary results}

We will obtain our results via the integral equation

$$
u(t)=\gamma(t) \alpha[u]+\int_{0}^{1} k(t, s) g(s) f(s, u(s)) d s:=\gamma(t) \alpha[u]+F u(t):=T u(t),
$$

where $\alpha[u]$ is as in (1.5). We want this integral operator to be well defined and compact in a cone $K$ in the space $C[0,1]$ of continuous functions endowed with the usual supremum norm. In order to define the new cone we use, we first list the standing hypotheses on the terms occurring in (2.1): 
$\left(C_{1}\right) k \geq 0$ is measurable, and for every $\tau \in[0,1]$ we have

$$
\lim _{t \rightarrow \tau}|k(t, s)-k(\tau, s)|=0 \text { for a.e. } s \in[0,1] .
$$

$\left(C_{2}\right)$ There exist a subinterval $[a, b] \subseteq[0,1]$, a measurable function $\Phi$, and a constant $c_{1} \in(0,1]$ such that

$$
\begin{aligned}
& k(t, s) \leq \Phi(s) \text { for } t \in[0,1] \text { and almost every } s \in[0,1] \\
& k(t, s) \geq c_{1} \Phi(s) \text { for } t \in[a, b] \text { and almost every } s \in[0,1] .
\end{aligned}
$$

$\left(C_{3}\right) \quad A$ is of bounded variation and $\mathcal{K}(s):=\int_{0}^{1} k(t, s) d A(t) \geq 0$ for a.e. $s$.

$\left(C_{4}\right) g \Phi \in L^{1}[0,1], g \geq 0$ a.e., and $\int_{a}^{b} \Phi(s) g(s) d s>0$.

$\left(C_{5}\right) \gamma \in C[0,1], \gamma(t) \geq 0,0 \leq \alpha[\gamma]<1$. We write $\Gamma:=\alpha[\gamma]$.

There exists $c_{2} \in(0,1]$ such that $\gamma(t) \geq c_{2}\|\gamma\|$ for $t \in[a, b]$.

$\left(C_{6}\right) f:[0,1] \times \mathbb{R}_{+} \rightarrow \mathbb{R}_{+}$satisfies Carathéodory conditions, that is, $f(\cdot, u)$ is measurable for each fixed $u \in \mathbb{R}_{+}$and $f(t, \cdot)$ is continuous for almost every $t \in[0,1]$, and for each $r>0$, there exists $\phi_{r} \in L^{\infty}[0,1]$ such that

$$
0 \leq f(t, u) \leq \phi_{r}(t) \text { for all } u \in[0, r] \text { and almost all } t \in[0,1] .
$$

The condition $\left(C_{6}\right)$ means that the singular behaviour of the nonlinearity is captured by the term $g$, a typical example being when the nonlinearity is $g(t) f(u)$ with $f$ continuous and $g \in L^{1}$. Note that $\left(C_{4}\right)$ implies that $g(s)>0$ on a subset of $[a, b]$ of positive measure but, in general, $g$ could be identically zero on some subinterval of $[0,1]$ and its singularities can occur at arbitrary points of $[0,1]$. Also, $\left(C_{1}\right),\left(C_{2}\right)$ and $\left(C_{4}\right)$ together imply that

$$
\begin{gathered}
\lim _{t \rightarrow \tau} \int_{0}^{1}|k(t, s)-k(\tau, s)| g(s) d s=0, \\
\lim _{t \rightarrow \tau} \int_{0}^{1}|k(t, s)-k(\tau, s)| g(s) \phi_{r}(s) d s=0,
\end{gathered}
$$

because each integrand is dominated by (a constant times) $2 \Phi(s) g(s)$. The 'continuity in $t$ ' assumption $\left(C_{1}\right)$ ensures that $\mathcal{K}(s)$ is defined for a.e. $s \in[0,1]$.

Let $P=\{u \in C[0,1]: u \geq 0\}$ denote the standard cone of nonnegative functions. Let $q: C[0,1] \rightarrow \mathbb{R}$ denote the continuous function

$$
q(u)=\min \{u(t): t \in[a, b]\},
$$


and let $c=\min \left\{c_{1}, c_{2}\right\}$ with $c_{1}$ as in $\left(C_{2}\right), c_{2}$ as in $\left(C_{5}\right)$. The new cone we use is defined as follows:

$$
K=\{u \in P, q(u) \geq c\|u\|, \alpha[u] \geq 0\} .
$$

Note that $\gamma \in K$ so $K \neq\{0\}$, and

$$
K=K_{0} \cap\{u \in P: \alpha[u] \geq 0\} \text {, where } K_{0}=\{u \in P, q(u) \geq c\|u\|\} .
$$

$K_{0}$ is a well-known type of cone, which has been used by, for example, D. Guo and Lakshmikantham [2], Krasnosel'skii and Zabreiko [9], and since used by many other authors in the study of multiple solutions of BVPs.

Remark 2.1 The condition $\left(C_{2}\right)$ was introduced in [11], it is not restrictive (see Theorem 2.2 below) and fits particularly well with use of the cone $K_{0}$ for many types of BVPs, including separated and nonlocal BCs. For example, when $k$ is continuous on $[0,1] \times[0,1]$ and $k(t, s)>0$ for $t \in(0,1), s \in[0,1]$ then $\left(C_{2}\right)$ holds for an arbitrary $[a, b] \subset(0,1)$. In fact we can take

$$
\Phi=\max _{(t, s) \in[0,1] \times[0,1]} k(t, s) \text { and } c_{1}=\min _{t \in[a, b], s \in[0,1]} k(t, s) / \Phi .
$$

The choice of $\Phi$ affects the choice of $c_{1}$ in $\left(C_{2}\right)$, and it can be useful to find a more precise function $\Phi$ and constant $c_{1}$.

We do not explicitly write the dependence on $[a, b]$, which is usually fixed, but simply write $K, q$ and $c$.

For $u \in P$, we define the maps $T, S$ by

$$
T u(t):=\gamma(t) \alpha[u]+\int_{0}^{1} k(t, s) g(s) f(s, u(s)) d s:=\gamma(t) \alpha[u]+F u(t)
$$

and

$$
\begin{aligned}
S u(t):=\frac{\gamma(t)}{1-\Gamma} \int_{0}^{1} \mathcal{K}(s) g(s) f(s, u(s)) d s & +\int_{0}^{1} k(t, s) g(s) f(s, u(s)) d s \\
& :=\int_{0}^{1} k_{S}(t, s) g(s) f(s, u(s)) d s .
\end{aligned}
$$

We also write $(2.7)$ in the form

$$
S u(t)=\frac{\gamma(t)}{1-\Gamma} \alpha[F u]+F u(t)
$$

We shall see below that $T, S$ are closely related, amongst other things $S$ and $T$ have the same fixed points in $K$. A direct derivation from the differential equation 
leads to a form of $S$ which looks different from (2.7). As an example, for the 3 -point $\mathrm{BCs} u^{\prime}(0)=0, u(1)=\alpha u(\eta), 0 \leq \alpha<1, T$ is given by

$$
T u(t)=\alpha u(\eta)+\int_{0}^{1} k(t, s) g(s) f(s, u(s)) d s, \text { where } k(t, s)=\left\{\begin{array}{l}
1-t \quad \text { if } s \leq t, \\
1-s \text { if } s>t .
\end{array}\right.
$$

and the direct derivation gives

$$
\begin{gathered}
S u(t)=\int_{0}^{1} k_{S}(t, s) g(s) f(s, u(s)) d s, \text { where } \\
k_{S}(t, s)=\frac{1}{1-\alpha}(1-s)-\left\{\begin{array}{ll}
\frac{\alpha}{1-\alpha}(\eta-s) & \text { if } s \leq \eta, \\
0 & \text { if } s>\eta,
\end{array}- \begin{cases}t-s & \text { if } s \leq t, \\
0 & \text { if } s>t .\end{cases} \right.
\end{gathered}
$$

but (2.7) would read, for this example,

$$
S u(t):=\frac{1}{1-\alpha} \int_{0}^{1} k(\eta, s) g(s) f(s, u(s)) d s+\int_{0}^{1} k(t, s) g(s) f(s, u(s)) d s,
$$

with $k$ as in (2.9). It can be verified that the two forms are equal. The form in (2.10) contains two negative terms (but the overall sum is positive) whereas (2.11) involves only positive terms.

Yet another advantage of our method is that $\left(C_{2}\right)$ is easily verified for $S$ because of the following result.

Theorem 2.2 If $k$ satisfies $\left(C_{1}\right),\left(C_{2}\right),\left(C_{3}\right),\left(C_{4}\right)$ and $\gamma$ satisfies $\left(C_{5}\right)$, then $k_{S}$ satisfies $\left(C_{1}\right),\left(C_{2}\right)$ for a function $\Phi_{1}$, the same interval $[a, b]$ and the same constant $c=\min \left\{c_{1}, c_{2}\right\}$, where $\Phi_{1}$ satisfies $\left(C_{4}\right)$.

Proof. We have

$$
\begin{aligned}
k_{S}(t, s) & =\frac{\gamma(t)}{1-\Gamma} \mathcal{K}(s)+k(t, s) \\
& \leq \frac{\|\gamma\|}{1-\Gamma} \mathcal{K}(s)+\Phi(s):=\Phi_{1}(s)
\end{aligned}
$$

and for $t \in[a, b]$,

$$
k_{S}(t, s) \geq \frac{c_{2}\|\gamma\|}{1-\Gamma} \mathcal{K}(s)+c_{1} \Phi(s) \geq \min \left\{c_{1}, c_{2}\right\} \Phi_{1}(s) .
$$

Note that $g \Phi_{1} \in L^{\infty}$ because $A$ has finite variation and $\mathcal{K}(s) \leq \Phi(s) \operatorname{Var}(A)$. 
Note that this result appears to be non obvious when one deals, for example, with the directly derived form of $k_{S}$ for $m$-point BVPs, e.g. [14]. In particular, it shows for the first time that for the standard well-studied $m$-point BVPs one can choose $[a, b]$ arbitrarily in $(0,1)$ so that $\left(C_{2}\right)$ holds.

Our integral operators are compact.

Lemma 2.3 Under the hypotheses $\left(C_{1}\right)-\left(C_{5}\right)$ the maps $T, S: P \rightarrow C[0,1]$ defined in (2.6), (2.7) are compact.

Proof. The compactness of $F$ follows from Proposition 3.1 of Chapter 5 of [18] since, as $[0,1]$ is compact, the limit in each of $(2.2),(2.3)$ is readily shown to be uniform in $\tau \in[0,1]$. Similarly $S$ is compact. The perturbation $\gamma(t) \alpha[u]$ is compact since it maps a bounded set into a bounded subset of a one dimensional space and thus $T$ is compact.

More importantly we show that $T$ and $S$ leave $K$ invariant, in fact $S$ maps $P$ into $K$.

Theorem 2.4 Under the hypotheses $\left(C_{1}\right)-\left(C_{5}\right), T: K \rightarrow K$ and $S: P \rightarrow K$.

Proof. To see that $T: K \rightarrow K$, for $u \in K$ and $t \in[0,1]$, we have,

$$
|T u(t)| \leq \alpha[u]|\gamma(t)|+\int_{0}^{1} k(t, s) g(s) f(s, u(s)) d s
$$

and so

$$
\|T u\| \leq \alpha[u]\|\gamma\|+\int_{0}^{1} \Phi(s) g(s) f(s, u(s)) d s .
$$

Also, for $t \in[a, b]$, we have

$$
T u(t) \geq c_{2} \alpha[u]\|\gamma\|+c_{1} \int_{0}^{1} \Phi(s) g(s) f(s, u(s)) d s \geq \min \left\{c_{1}, c_{2}\right\}\|T u\| .
$$

Using Theorem 2.2, a similar calculation works for $S$ with $u \in P$. Now we need to show that $\alpha[T u] \geq 0$ for $u \in K$ and that $\alpha[S u] \geq 0$ for $u \in P$. Firstly we have, using $\left(C_{3}\right)$, for $u \in P$,

$$
\alpha[F u]=\int_{0}^{1} \int_{0}^{1} k(t, s) g(s) f(s, u(s)) d s d A(t)=\int_{0}^{1} \mathcal{K}(s) g(s) f(s, u(s)) d s \geq 0 .
$$

Therefore, for $u \in K$ we have,

$$
\alpha[T u]=\alpha[\gamma] \alpha[u]+\alpha[F u] \geq 0 .
$$


Secondly, from (2.8), for $u \in P$ we have

$$
\alpha[S u]=\frac{\Gamma}{1-\Gamma} \alpha[F u]+\alpha[F u] \geq 0 .
$$

Lemma 2.5 $S$ and $T$ have the same fixed points (in $K$ ).

Proof. Suppose that $u(t)=S u(t)$. Then applying the linear functional $\alpha$ using (2.8) we obtain

$$
\alpha[u]=\frac{\Gamma}{1-\Gamma} \alpha[F u]+\alpha[F u]=\frac{1}{1-\Gamma} \alpha[F u]
$$

Substituting into $u(t)=S u(t)$ gives $u(t)=\gamma(t) \alpha[u]+F u(t)=T u(t)$. The converse is proved similarly.

Let $D$ be a bounded open set in $C[0,1]$ and write $D_{K}$ for $D \cap K$ so that $D$ is open in $K$ and let $\partial D_{K}$ denote the boundary of $K$ (in the relative topology). We use standard properties of the classical fixed point index $i_{K}\left(T, D_{K}\right)$ for a compact map $T$, see for example [1] or [2] for further information. We begin with a very useful observation.

Theorem 2.6 If $u \neq T u$ for $u \in \partial D_{K}$, then $i_{K}\left(T, D_{K}\right)=i_{K}\left(S, D_{K}\right)$.

Proof. By Lemma 2.5, both indices are defined. By the homotopy property it suffices to prove that

$$
u \neq \beta T u+(1-\beta) S u, \text { for all } \beta \in[0,1] \text { and for all } u \in \partial D_{K} .
$$

Suppose this fails, that is there exist $u \in \partial D_{K}$ and $\beta \in[0,1]$ such that

$$
u(t)=\beta T u(t)+(1-\beta) S u(t) .
$$

Applying $\alpha$ gives

$$
\alpha[u]=\beta(\Gamma \alpha[u]+\alpha[F u])+(1-\beta)\left(\frac{\Gamma}{1-\Gamma} \alpha[F u]+\alpha[F u]\right) .
$$

Hence we have

$$
(1-\beta \Gamma) \alpha[u]=\beta \alpha[F u]+\frac{1-\beta}{1-\Gamma} \alpha[F u]
$$

so that $\alpha[u]=\frac{1}{1-\Gamma} \alpha[F u]$. This leads to $u(t)=\frac{\gamma(t)}{1-\Gamma} \alpha[F u]+F u(t)=S u(t)$, a contradiction. 
This result means that we will obtain the same fixed point index results whichever representation we use. However, we believe there is an advantage in using the form involving $T$ rather than $S$. In particular, it allows us to treat $m$-point BVPs with coefficients $\alpha_{i}$ of either sign and we easily get conditions on these coefficients which ensure the kernel is positive, and which do not seem to be obvious from the explicitly derived form of $S$, for example, a more complicated version of (2.10), as in [14].

\section{$3 \quad$ Fixed point index calculations}

For $\rho>0$ we define the following open subsets of $K$ :

$$
K_{\rho}:=\{u \in K:\|u\|<\rho\}, \quad V_{\rho}:=\{u \in K: q(u)<\rho\} .
$$

The set $V_{\rho}$ was essentially introduced in [10], in fact $V_{\rho}=\Omega_{\rho / c}$ using the notation of [10]. We believe that using $V_{\rho}$ is natural and makes it clear that choosing $c$ as large as possible yields a weaker condition to be satisfied by $f$ in the following result.

Theorem 3.1 1. Suppose that $f(t, u) \leq \rho f^{0, \rho}$ for $t \in[0,1]$ and $0 \leq u \leq \rho$. Then we have $i_{K}\left(T, K_{\rho}\right)=1$ if

$$
f^{0, \rho}\left(\sup _{t \in[0,1]}\left\{\frac{\gamma(t)}{1-\Gamma} \int_{0}^{1} \mathcal{K}(s) g(s) d s+\int_{0}^{1} k(t, s) g(s) d s\right\}\right)<1 .
$$

2. Suppose that $f(t, u) \geq \rho f_{\rho, \rho / c}$ for $t \in[a, b]$ and $\rho \leq u \leq \rho / c$. Then we have $i_{K}\left(T, V_{\rho}\right)=0$ if

$$
f_{\rho, \rho / c}\left(\inf _{t \in[a, b]}\left\{\frac{\gamma(t)}{1-\Gamma} \int_{a}^{b} \mathcal{K}(s) g(s) d s+\int_{a}^{b} k(t, s) g(s) d s\right\}\right)>1 .
$$

Proof. (1) We show that $T u \neq \lambda u$ for all $\lambda \geq 1$ when $u \in \partial K_{\rho}$, which implies that $i_{K}\left(T, K_{\rho}\right)=1$. In fact, if not, then there exist $u$ with $\|u\|=\rho$ and $\lambda \geq 1$ such that

$$
\lambda u(t)=T u(t)=\gamma(t) \alpha[u]+F u(t) .
$$

Therefore $\lambda \alpha[u]=\Gamma \alpha[u]+\alpha[F u]$ and hence $\lambda u(t)=\frac{\gamma(t)}{\lambda-\Gamma} \alpha[F u]+F u(t)$.

Using the inequality $f(s, u(s)) \leq \rho f^{0, \rho}$ and taking the supremum over $[0,1]$ gives

$$
\lambda \rho \leq \rho f^{0, \rho}\left(\sup _{t \in[0,1]}\left\{\frac{\gamma(t)}{1-\Gamma} \int_{0}^{1} \mathcal{K}(s) g(s) d s+\int_{0}^{1} k(t, s) g(s) d s\right\}\right)
$$

contradicting (3.1). 
(2) We show that $u \neq T u+\lambda \gamma$ for all $\lambda \geq 0$ and $u \in \partial V_{\rho}$ which implies that $i_{K}\left(T, V_{\rho}\right)=0$. In fact, if there is $u \in \partial V_{\rho}$ and $\lambda \geq 0$ with $u=T u+\lambda \gamma=$ $\gamma \alpha[u]+F u+\lambda \gamma$, then $\alpha[u]=\Gamma \alpha[u]+\alpha[F u]+\lambda \Gamma$. Therefore we have

$$
u(t)=\frac{\gamma(t)}{1-\Gamma}(\alpha[F u]+\lambda \Gamma)+F u(t)+\lambda \gamma(t)=\frac{\gamma(t)}{1-\Gamma} \alpha[F u]+F u(t)+\frac{\lambda}{1-\Gamma} \gamma(t) .
$$

We replace the integrals over $[0,1]$ by integrals over $[a, b]$, note that, for $u \in \partial V_{\rho}$, $q(u)=\rho$ and $\rho \leq u(s) \leq \rho / c$ for $s \in[a, b]$, and use the inequality $f(s, u(s)) \geq$ $\rho f_{\rho, \rho / c}$. Taking the infimum for $t \in[a, b]$ then gives

$$
\rho=q(u) \geq \rho f_{\rho, \rho / c}\left(\inf _{t \in[a, b]}\left\{\frac{\gamma(t)}{1-\Gamma} \int_{a}^{b} \mathcal{K}(s) g(s) d s+\int_{a}^{b} k(t, s) g(s) d s\right\}\right),
$$

for $\lambda \geq 0$, contradicting $(3.2)$.

Remark 3.2 These two results can be written (loosely) in the following forms

$$
f^{0, \rho}<m_{S} \Longrightarrow i_{K}\left(T, K_{\rho}\right)=1 ; f_{\rho, \rho / c}>M_{S}(a, b) \Longrightarrow i_{K}\left(T, V_{\rho}\right)=0 .
$$

Here

$$
\frac{1}{m_{S}}:=\sup _{t \in[0,1]} \int_{0}^{1} k_{S}(t, s) g(s) d s ; \frac{1}{M_{S}(a, b)}:=\inf _{t \in[a, b]} \int_{a}^{b} k_{S}(t, s) g(s) d s .
$$

These could be deduced from known results for $S$, for example [21], since $S$ satisfies the appropriate hypotheses as is shown in Theorem 2.2, and then using Theorem 2.6. We have given the direct proofs because often $[a, b]$ can be chosen arbitrarily in $(0,1)$. In this case an obvious question is whether there is some optimal choice. For a given $[a, b],(3.2)$ shows that we should choose $c=c(a, b)$ as large as possible. We also have a weaker restriction on $f$ when $M_{S}(a, b)$ is as small as possible. In some simple cases, determining $[a, b]$ so as to minimize $M_{S}(a, b)$ can be achieved, see [19], but the calculations quickly become tricky, see [20]. However, using our method, we get good choices that can be calculated more easily. These are the following.

Theorem 3.3 1. Suppose $f(t, u) \leq \rho f^{0, \rho}$ for $t \in[0,1]$ and $0 \leq u \leq \rho$. Then we have $i_{K}\left(T, K_{\rho}\right)=1$ if

$$
\begin{gathered}
f^{0, \rho}\left(\frac{\|\gamma\|}{1-\Gamma} \int_{0}^{1} \mathcal{K}(s) g(s) d s+\frac{1}{m}\right)<1, \\
\text { where } \frac{1}{m}=\sup _{t \in[0,1]} \int_{0}^{1} k(t, s) g(s) d s .
\end{gathered}
$$


2. Suppose $f(t, u) \geq \rho f_{\rho, \rho / c}$ for $t \in[a, b]$ and $\rho \leq u \leq \rho / c$. Then we have $i_{K}\left(T, V_{\rho}\right)=0$ if

$$
\begin{gathered}
f_{\rho, \rho / c}\left(\frac{c_{2}\|\gamma\|}{1-\Gamma} \int_{a}^{b} \mathcal{K}(s) g(s) d s+\frac{1}{M(a, b)}\right)>1 . \\
\text { where } \frac{1}{M(a, b)}:=\inf _{t \in[a, b]} \int_{a}^{b} k(t, s) g(s) d s .
\end{gathered}
$$

Remark 3.4 $M(a, b)$ corresponds to a simpler kernel than does $M_{S}(a, b)$ so determining the interval $[a, b]$ which makes $M(a, b)$ minimal is also simpler.

Webb and Lan [21] gave criteria for the existence of (multiple) positive solutions of Hammerstein integral operators of the form $u(t)=S u(t)$ which depend on the relationship between the behaviour of $f(t, u) / u$ as $u$ tends to $0^{+}$or $\infty$ and the principal (smallest positive) characteristic value $\mu_{1}$ of an associated linear integral operator. An example is replacing the conditions

$$
f_{\rho_{1}, \rho_{1} / c}>M_{S}(a, b), \quad f^{0, \rho_{2}}<m_{S}, \text { for } \rho_{1} \text { small and } \rho_{2} \text { large, }
$$

by

$$
\liminf _{u \rightarrow 0+} f(u) / u>\mu_{1}, \quad \limsup _{u \rightarrow \infty} f(u) / u<\mu_{1},
$$

which gives an optimal result. This uses some fixed point index calculations from [21]. It was shown in [21] that $m_{S} \leq \mu_{1} \leq M_{S}(a, b)$ and the inequalities are strict if the corresponding eigenfunction is not constant, so using (3.8) usually gives a better result than using (3.7). This does not make the constants $m_{S}, M_{S}$ redundant, because they are useful in taking account of the behaviour of $f(t, u)$ with respect to $u$ on bounded intervals not containing 0 , and are used explicitly in results concerning the existence of multiple solutions, see Theorem 4.2.

We will use some results from [21] which apply to $S$ to deduce results for $T$. We first define the linear operator $L_{S}$ corresponding to $S$. We define

$$
L_{S}(u):=\int_{0}^{1} k_{S}(t, s) g(s) u(s) d s .
$$

Similar to the proofs of Lemma 2.3 and Theorem 2.4, $L_{S}$ is compact and maps $P$ into $K$. We recall that $\lambda$ is an eigenvalue of an operator $L$ with corresponding eigenfunction $\varphi$ if $\varphi \neq 0$ and $\lambda \varphi=L \varphi$. The reciprocals of nonzero eigenvalues are called characteristic values of $L$. The characteristic values of the integral operator are often called eigenvalues of the differential operator from which they arise.

Theorem 3.5 When $\left(C_{1}\right)-\left(C_{4}\right)$ hold, $r\left(L_{S}\right)$, the radius of the spectrum of $L_{S}$, is an eigenvalue of $L_{S}$ with eigenfunction in $K$. 
Proof. This is a consequence of the Krein-Rutman theorem which shows that $r\left(L_{S}\right)$ is an eigenvalue of $L_{S}$ with eigenfunction in $P .\left(C_{4}\right)$ is used to show $r\left(L_{S}\right)>0$, see [21]. As $L_{S}$ maps $P$ into $K$, the eigenfunction belongs to $K$.

We note that $\mu_{1}:=1 / r\left(L_{S}\right)$ satisfies $\varphi=\mu_{1} L_{S} \varphi$ if and only if

$$
\varphi=\gamma(t) \alpha[\varphi]+\mu_{1} L_{F} \varphi
$$

where $L_{F} u=\int_{0}^{1} k(t, s) g(s) u(s) d s$, by the same calculation as in the proof of Lemma 2.5. Equation (3.9) tells us how we must define $L_{T}$ and 'eigenvalues' of $L_{T}$.

We also define an operator

$$
\tilde{L}_{S}:=\int_{0}^{1} \tilde{k}_{S}(t, s) g(s) u(s) d s:=\int_{a}^{b} k_{S}(t, s) g(s) u(s) d s .
$$

Since the kernel $\tilde{k}_{S}$ satisfies the same hypotheses as $k_{S}, r(\tilde{L})$ is an eigenvalue of $\tilde{L}_{S}$ with eigenfunction in $K$. Let $\tilde{\mu}_{1}=1 / r\left(\tilde{L}_{S}\right)$. We shall use the following concept.

Definition 3.6 We say that $L$ satisfies $(U P E)$ if $r(L)$ is the only positive eigenvalue of $L$ with an eigenfunction in the cone $P$.

$(U P E)$ holds for $L_{S}$ whenever $k_{S}$ is symmetric: $k_{S}(t, s)=k_{S}(s, t)$, and $g \in L^{1}$, see [21]; but the kernel need not by symmetric. Also $(U P E)$ holds if $L_{S}$ is strongly positive, that is $L_{S}$ maps $P \backslash\{0\}$ into the interior of $P$, by Theorem 3.2 of [1] but, for BVPs with the BC $u(0)=0$ the corresponding $L_{S}$ is not strongly positive since $L_{S} u(0)=0$. It is often possible to verify $(U P E)$ when $g \equiv 1$ directly from the differential equation, see [20, 21].

Notation We make the following definitions.

$$
\begin{aligned}
\bar{f}(u): & =\sup _{t \in[0,1]} f(t, u), \underline{f}(u):=\inf _{t \in[0,1]} f(t, u) ; \\
f^{0} & =\limsup _{u \rightarrow 0+} \bar{f}(u) / u, \quad f_{0}=\liminf _{u \rightarrow 0+} \underline{f}(u) / u ; \\
f^{\infty} & =\limsup _{u \rightarrow \infty} \bar{f}(u) / u, \quad f_{\infty}=\liminf _{u \rightarrow \infty} \underline{f}(u) / u .
\end{aligned}
$$

The following result collects together some results, proved in [21] for operators of the form $S$, and gives conditions which ensure the fixed point index of $T$ on suitable subsets of $K$ is either 0 or 1 . These can then be used in a standard way to get multiplicity results; we state some of these below.

Theorem 3.7 (1) If $0 \leq f^{0}<\mu_{1}$, then there exists $\rho_{0}>0$ such that

$$
i_{K}\left(T, K_{\rho}\right)=1 \text { for each } \rho \in\left(0, \rho_{0}\right] .
$$


(2) If $0 \leq f^{\infty}<\mu_{1}$, then there exists $R_{0}$ such that

$$
i_{K}\left(T, K_{R}\right)=1 \text { for each } R>R_{0}
$$

(3) If $\mu_{1}<f_{0} \leq \infty$, then there exists $\rho_{0}>0$ such that for each $\rho \in\left(0, \rho_{0}\right]$, if $u \neq T u$ for $u \in \partial K_{\rho}$, then

$$
i_{K}\left(T, K_{\rho}\right)=0 \text {. }
$$

(4) If $\tilde{\mu}_{1}<f_{\infty} \leq \infty$, then there exists $R_{1}$ such that for each $R \geq R_{1}$, if $u \neq T u$ for $u \in \partial K_{R}$, then

$$
i_{K}\left(T, K_{R}\right)=0 .
$$

$\left(4^{\prime}\right)$ Suppose also that $L$ satisfies $(U P E)$ and that $\left(C_{2}\right)$ holds for an arbitrary $[a, b] \subset(0,1)$. If $\mu_{1}<f_{\infty} \leq \infty$, then there exists $R_{1}$ such that for each $R \geq R_{1}$, if $u \neq T u$ for $u \in \partial K_{R}$, then

$$
i_{K}\left(T, K_{R}\right)=0
$$

Proof. The results of [21] apply to $S$ and give the above statements with $S$ in place of $T$, we then apply Theorem 2.6.

Note that $\tilde{\mu}_{1} \geq \mu_{1}$ so $\left(4^{\prime}\right)$ gives a stronger result than (4). When $\left(4^{\prime}\right)$ holds, the index $i_{K}\left(T, K_{R}\right)=0$ is independent of $[a, b]$ (recall that $K$ depends on $[a, b]$ ) so we can use any fixed $[a, b]$ that is convenient for other purposes.

\section{Existence of multiple positive solutions of inte- gral equations}

We use the notation of the previous section and state results on existence of multiple nonzero positive solutions for the equation

$$
u(t)=T u(t):=\gamma(t) \alpha[u]+\int_{0}^{1} k(t, s) g(s) f(s, u(s)) d s, \quad t \in[0,1] .
$$

We say that $\left(C_{2}^{*}\right)$ holds if $\left(C_{2}\right)$ is valid for an arbitrary interval $[a, b] \subset(0,1)$. However our theorems are for a fixed $[a, b]$ and corresponding cone $K$.

Theorem 4.1 Assume that $\left(C_{1}\right)-\left(C_{6}\right)$ hold and that one of the following conditions holds.

$\left(H_{1}\right) 0 \leq f^{0}<\mu_{1}$ and $\tilde{\mu}_{1}<f_{\infty} \leq \infty$.

$\left(H_{2}\right) 0 \leq f^{\infty}<\mu_{1}$ and $\mu_{1}<f_{0} \leq \infty$.

Then (4.1) has a positive solution $u \in K, \rho \leq\|u\| \leq R$ for some $0<\rho<R$. When $L_{S}$ satisfies $(U P E)$ and $\left(C_{2}^{*}\right)$ holds, we may replace $\tilde{\mu}_{1}$ by $\mu_{1}$ in $\left(H_{1}\right)$. 
The proof is by applying Theorem 3.7 on $K_{\rho}$ and $K_{R}$ for $\rho$ sufficiently small and $R$ sufficiently large and using the additivity property of fixed point index. This gives some optimal results and is a real improvement on the earlier ones where, for example, in the second case it was assumed that

$$
0 \leq f^{\infty}<m_{S}, \quad M_{S}<f_{0} \leq \infty .
$$

We also state results for the existence of at least two and at least three (strictly) positive solutions.

Theorem 4.2 Assume that $\left(C_{1}\right)-\left(C_{6}\right)$ hold. Then (4.1) has two positive solutions in $K$ if one of the following conditions holds.

$\left(S_{1}\right) 0 \leq f^{0}<\mu_{1}, f_{\rho, \rho / c}>M_{S}$ for some $\rho>0$, and $0 \leq f^{\infty}<\mu_{1}$.

$\left(S_{2}\right) \mu_{1}<f_{0} \leq \infty, f^{0, \rho}<m_{S}$ for some $\rho>0$, and $\tilde{\mu}_{1}<f_{\infty} \leq \infty$.

Equation (4.1) has at least three positive solutions if either $\left(T_{1}\right)$ or $\left(T_{2}\right)$ below holds.

$\left(T_{1}\right)$ There exist $0<\rho_{1}<\rho_{2}<\infty$, such that

$$
\mu_{1}<f_{0} \leq \infty, \quad f^{0, \rho_{1}}<m_{S}, \quad f_{\rho_{2}, \rho_{2} / c}>M_{S}, \quad 0 \leq f^{\infty}<\mu_{1} .
$$

$\left(T_{2}\right)$ There exist $0<\rho_{1}<c \rho_{2}<\infty$, such that

$$
0 \leq f^{0}<\mu_{1}, \quad f_{\rho_{1}, \rho_{1} / c}>M_{S}, f^{0, \rho_{2}}<m_{S}, \quad \tilde{\mu}_{1}<f_{\infty} \leq \infty .
$$

When $L_{S}$ satisfies $(U P E)$ and $\left(C_{2}^{*}\right)$ holds, we may replace $\tilde{\mu}_{1}$ by $\mu_{1}$.

The proof is an application of the additivity property of fixed point index, see [21] for details. It is possible to give criteria for the existence of a countable number of positive solutions by extending the list of conditions. We omit the routine statement of such results.

\section{Boundary Value problems}

\subsection{The BCs (1.2)}

We first study the BVP

$$
\begin{aligned}
-u^{\prime \prime}(t) & =g(t) f(t, u(t)), t \in(0,1), \\
u(0) & =0, u(1)=\alpha[u],
\end{aligned}
$$

where $\alpha[u]=\int_{0}^{1} u(s) d A(s)$ for a signed measure $d A$. Special cases of these BCs have been studied by Ma [15] and Karakostas and Tsamatos [7]. Ma studied the problem

$$
-u^{\prime \prime}(t)=g(t) f(u(t)), u(0)=0, u(1)=\int_{\alpha}^{\beta} h(s) u(s) d s,
$$


with $g \geq 0, f \geq 0$ both continuous and with $0 \leq \beta \int_{\alpha}^{\beta} h(s) d s<1$, where $0<\alpha<$ $\beta<1$, which implies $\int_{\alpha}^{\beta} t h(t) d t<1$. Here $h \geq 0$ is continuous and is 0 outside $[\alpha, \beta]$, and $g$ is strictly positive at some point of $[\beta, 1]$. Karakostas and Tsamatos [7] studied the same equation with the same assumptions except the $\mathrm{BC}$ has the more general form

$$
u(1)=\int_{\alpha}^{\beta} u(s) d A(s)
$$

where $d A$ is supported on $[\alpha, \beta], A$ is increasing, (corresponding to a (positive) measure), with the requirement $\beta \int_{\alpha}^{\beta} d A(t)<1$. Both authors made this last assumption in order to relate their problem to a 3-point problem, our method does not need knowledge of an earlier problem but can be used to deduce results for 3-point problems. By applying our result we shall see that the assumptions can be weakened: $g$ need not be continuous, the measure $d A$ can be a signed measure, and it does not have to be supported on some subinterval $[\alpha, \beta]$. We also use better conditions on $f$. For a signed measure we show below that we need

$$
0 \leq \int_{0}^{1} t d A(t)<1
$$

together with

$$
(1-s) \int_{0}^{s} t d A(t)+s \int_{s}^{1}(1-t) d A(t) \geq 0, \text { for a.e. } s \in[0,1] .
$$

This clearly strictly includes the case studied by Karakostas and Tsamatos [7] and Ma [15] since (5.6) is trivially satisfied when $d A$ is a positive measure. We give explicit examples of sign changing measures that satisfy our requirements below. In the discrete sign changing case we obtain conditions for existence of positive solutions of $m$-point BVPs which are close to necessary.

Our existence and multiplicity results of section 4 require less stringent restrictions on $f$ than $[7,15]$. Ma only considered the case when $f$ is either sub- or super-linear, that is, either $f_{0}=\infty$ and $f^{\infty}=0$ or $f^{0}=0$ and $f_{\infty}=\infty$, respectively and obtained the existence of one positive solution. Karakostas and Tsamatos gave results for the existence of one, two or three positive solutions. Our conditions on $f$ are weaker because firstly we use the constants $m_{S}, M_{S}$ which are better than those used in [7] [two negative terms are discarded in a calculation in [7]], and secondly we also use $\mu_{1}$ which gives even sharper results. As noted above, we could easily state results for countably many solutions too.

For the BCs (1.2) the operator $T$ is

$$
T u(t)=t \alpha[u]+\int_{0}^{1} k(t, s) g(s) f(s, u(s)) d s, \text { where } k(t, s)= \begin{cases}s(1-t) & \text { if } s \leq t, \\ t(1-s) & \text { if } s>t .\end{cases}
$$


We take $[a, b]=[1 / 4,3 / 4]$. It is easy to check that $k(t, s) \leq s(1-s):=\Phi(s)$ and that $\min _{t \in[1 / 4,3 / 4]} k(t, s) \geq(1 / 4) s(1-s)$, that is, $c_{1}=1 / 4$ for this choice of $[a, b]$. Also it is clear that $c_{2}=1 / 4$. In fact we have chosen $[1 / 4,3 / 4]$ because it gives the minimal $M$ in the conditions of Theorem 3.3 , but since $k(t, s)$ written in (5.7) satisfies $\left(C_{2}^{*}\right)$ the choice of $[a, b]$ is at our disposal. Now let $A$ be of bounded variation. To verify $\left(C_{3}\right)$ we have to show that

$$
\mathcal{K}(s)=\int_{0}^{1} k(t, s) d A(t) \geq 0
$$

Thus we want

$$
(1-s) \int_{0}^{s} t d A(t)+s \int_{s}^{1}(1-t) d A(t) \geq 0 .
$$

This is as claimed above in (5.6). The condition $0 \leq \Gamma<1$ is (5.5). We do not state the obvious theorems which follow directly by applying the results of section 4 .

We give an explicit example in the continuously distributed case.

Example 5.1 Let $\alpha(t)=-\cos (2 \pi t)$. Then $d A(t):=\alpha(t) d t$ changes sign on $[0,1]$ and satisfies (5.8).

In fact, by integration by parts,

$$
(1-s) \int_{0}^{s} t d A(t)+s \int_{s}^{1}(1-t) d A(t)=s \int_{0}^{1} A(t) d t-\int_{0}^{s} A(t) d t
$$

where $A(t)=\int_{0}^{t} d A(s)$. In our example this is

$$
s\left(\frac{\cos (2 \pi)-1}{4 \pi^{2}}\right)+\frac{1-\cos (2 \pi s)}{4 \pi^{2}}=\frac{1-\cos (2 \pi s)}{4 \pi^{2}} \geq 0 .
$$

Also we have

$$
\Gamma=\int_{0}^{1} t d A(t)=\int_{0}^{1}-t \cos (2 \pi t) d t=0
$$

This means we have verified all the hypotheses, so Theorem 4.2 can be applied once the constants have been calculated. Of course, we can obtain many more examples of the form $h(t)-\cos (2 \pi t)$, where $h(t) \geq 0$ and $\int_{0}^{1} t h(t) d t<1$.

Example 5.2 A discrete (4-point) problem. Consider the BC

$$
u(0)=0, u(1)=\alpha_{1} u\left(\eta_{1}\right)+\alpha_{2} u\left(\eta_{2}\right), \text { where } 0<\eta_{1}<\eta_{2}<1 .
$$

We will determine the restrictions to be placed on $\alpha_{1}, \alpha_{2}$ so that our hypotheses are satisfied. 
We have $\alpha[u]=\alpha_{1} u\left(\eta_{1}\right)+\alpha_{2} u\left(\eta_{2}\right)$. Then $\Gamma=\alpha_{1} \eta_{1}+\alpha_{2} \eta_{2}$ so we want

$$
0 \leq \alpha_{1} \eta_{1}+\alpha_{2} \eta_{2}<1
$$

Also $\mathcal{K}(s)=\alpha_{1} k\left(\eta_{1}, s\right)+\alpha_{2} k\left(\eta_{2}, s\right)$. Thus for $\mathcal{K}(s) \geq 0$ we need

$$
\begin{aligned}
& \alpha_{1} s\left(1-\eta_{1}\right)+\alpha_{2} s\left(1-\eta_{2}\right) \geq 0 \text { for } 0 \leq s \leq \eta_{1} \\
& \alpha_{1} \eta_{1}(1-s)+\alpha_{2} s\left(1-\eta_{2}\right) \geq 0 \text { for } \eta_{1}<s \leq \eta_{2} \\
& \alpha_{1} \eta_{1}(1-s)+\alpha_{2} \eta_{2}(1-s) \geq 0 \text { for } s>\eta_{2} .
\end{aligned}
$$

The total requirement is therefore

$$
\begin{aligned}
& 0 \leq \alpha_{1} \eta_{1}+\alpha_{2} \eta_{2}<1, \text { and } \\
& \alpha_{1}\left(1-\eta_{1}\right)+\alpha_{2}\left(1-\eta_{2}\right) \geq 0 .
\end{aligned}
$$

This defines a region in the $\alpha_{1}, \alpha_{2}$-plane which is much larger than the 'obvious' region defined by

$$
\alpha_{1} \geq 0, \alpha_{2} \geq 0,0 \leq \alpha_{1} \eta_{1}+\alpha_{2} \eta_{2}<1 \text {. }
$$

The conditions (5.10), (5.11) were given by a much longer method in [5] working directly with the explicit directly derived form of the kernel $k_{S}$. Applying our method developed here, we can easily write down the conditions for the general $m$-point problem, but we do not list these. Positivity of the coefficients $\alpha_{i}$, as in (5.12), was used in [14] where the existence of one positive solution was shown when $f$ is either sub- or super-linear, and in [22] where one or two positive solutions was obtained using hypotheses on $f$ similar to, but more restrictive than, those in the first part of Theorem 4.2. A result for three positive solutions has been given in [13] (but one of these might be zero), by a direct, more complicated method, again assuming the coefficients are positive and using conditions on $f$ stronger than those in $\left(S_{1}\right)$ in Theorem 4.2. Sign changing coefficients have been allowed previously for $m$-point problems, for example in [4], but not for existence of positive solutions.

For this problem the constants can be calculated, when $g \equiv 1$ the eigenvalue can be easily calculated numerically, e.g. by Maple. But it is not clear whether (UPE) holds; this was shown in [21] for the $m$-point problem when all the $\alpha_{i}$ are positive, as in (5.12), but the sign changing case remains open.

\subsection{The BCs (1.3)}

We briefly indicate how our results apply to these BCs and give an example of the numbers that arise in the continuously distributed case. This BC has been studied extensively in the 3-point case, references may be found in $[7,14,19]$. A more general set of BCs for $m$-point BVPS has been studied in $[12,16]$, the former allowing singularities in $f$ in the $u$ variable. Our general approach gives 
completely new results for both $m$-point and integral BCs without the type of calculation seen in these papers. For this BC, $\gamma \equiv 1$ and

$$
k(t, s)= \begin{cases}1-t, & \text { if } s \leq t, \\ 1-s, & \text { if } s>t .\end{cases}
$$

For this kernel, $[a, b]$ may be chosen arbitrarily in $[0,1)$. The condition $\mathcal{K}(s) \geq 0$ now means

$$
(1-s) \int_{0}^{s} d A(t)+\int_{s}^{1}(1-t) d A(t)=\int_{s}^{1} A(t) d t \geq 0 .
$$

Some examples of sign changing measures that lead to $\mathcal{K}(s) \geq 0$ are given by $\alpha_{1}(t)=\sin (2 \pi t)$ with $\Gamma_{1}=0$ and $\alpha_{2}(t)=\cos (\pi t)$ with $\Gamma_{2}=0$. For the 4-point problem

$$
u^{\prime}(0)=0, \quad u(1)=\alpha[u]:=\alpha_{1} u\left(\eta_{1}\right)+\alpha_{2} u\left(\eta_{2}\right), \text { where } 0<\eta_{1}<\eta_{2}<1,
$$

we want

$$
0 \leq \Gamma<1, \text { that is } 0 \leq \alpha_{1}+\alpha_{2}<1 .
$$

For $\mathcal{K}(s) \geq 0$, as in Lemma 5.1 we obtain the conditions

$$
\alpha_{1}\left(1-\eta_{1}\right)+\alpha_{2}\left(1-\eta_{2}\right) \geq 0, \quad \alpha_{1}+\alpha_{2} \geq 0 .
$$

The total requirement is

$$
0 \leq \alpha_{1}+\alpha_{2}<1, \quad \alpha_{1}\left(1-\eta_{1}\right)+\alpha_{2}\left(1-\eta_{2}\right) \geq 0,
$$

again a much larger region than the part in the first quadrant. These conditions were found in [5] by a longer calculation. A direct application of the results of Section 4 give improvements on those in [5], while other authors have assumed positivity of the coefficients.

Example 5.3 Consider the differential equation with $g \equiv 1$ and with the BCs $u^{\prime}(0)=0, u(1)=\int_{0}^{1} \alpha u(s) d s$, where $\alpha$ is a constant.

Then $\Gamma=\alpha$ so we need $0 \leq \alpha<1$. Also, we have

$$
\begin{aligned}
\mathcal{K}(s) & =\int_{0}^{1} \alpha k(t, s) d t=\alpha\left(\int_{0}^{s}(1-s) d t+\int_{s}^{1}(1-t) d t\right) \\
& =\frac{\alpha}{2}\left(1-s^{2}\right) \geq 0 .
\end{aligned}
$$

We use the conditions (and the notation) of Theorem 3.3. Since $t \mapsto k(t, s)$ is decreasing, we have

$$
\frac{1}{m}=\max _{t \in[0,1]} k(t, s) d s=\int_{0}^{1} k(0, s) d s=\frac{1}{2} .
$$




$$
\frac{1}{M(a, b)}=\min _{t \in[a, b]} k(t, s) d s=\int_{a}^{b} k(b, s) d s=(b-a)(1-b) .
$$

$M(a, b)$ is minimized by taking $a=0, b=1 / 2$ and $M(0,1 / 2)=4$. For this choice of $[a, b], \Phi(s)=1-s$ and $c_{1}=1 / 2, c_{2}=1$. The conditions (3.5), (3.6) now read

$$
\begin{aligned}
& f^{0, \rho}\left(\frac{\alpha}{1-\alpha} \frac{1}{3}+\frac{1}{2}\right) \leq 1, \\
& f_{\rho, \rho / c}\left(\frac{\alpha}{1-\alpha} \frac{11}{48}+\frac{1}{4}\right) \geq 1 .
\end{aligned}
$$

For example, taking $\alpha=1 / 2$ we get the numbers

$$
f^{0, \rho} \leq 6 / 5=1.2, \quad f_{\rho, \rho / c} \geq 48 / 23 \simeq 2.087 .
$$

The 'eigenvalue' $\mu_{1}$ is found by looking for solutions of $-u^{\prime \prime}=\omega^{2} u$. The nonzero solutions are $\cos (\omega t)$ and $\mu_{1}=\omega^{2}$, where $\omega$ is the smallest positive root of the equation

$$
\cos (\omega)=\int_{0}^{1} \alpha \cos (\omega t) d t=\alpha \sin (\omega) / \omega .
$$

When $\alpha=1 / 2$, we find, using Maple, that $\mu_{1} \simeq 1.359$ (rounded to 3 decimal places). This ties in with our general assertion that $m_{S}<\mu_{1}<M_{S}$ (for a nonconstant eigenfunction) and shows that using $\mu_{1}$ gives stronger results.

\subsection{The BCs (1.4)}

For this $\mathrm{BC}, \gamma(t)=t$ and

$$
k(t, s)= \begin{cases}s, & \text { if } s \leq t \\ t, & \text { if } s>t\end{cases}
$$

In this case $[a, b]$ may be chosen arbitrarily in $(0,1]$. The condition $\mathcal{K}(s) \geq 0$ means

$$
\int_{0}^{s} t d A(t)+\int_{s}^{1} s d A(t) \geq 0 .
$$

An example of a sign changing measure that gives $\mathcal{K}(s) \geq 0$ is $\alpha(t)=-\cos (\pi t)$ with $\Gamma=2 / \pi^{2}$.

For the 4-point problem

$$
u(0)=0, u^{\prime}(1)=\alpha[u]:=\alpha_{1} u\left(\eta_{1}\right)+\alpha_{2} u\left(\eta_{2}\right), \text { where } 0<\eta_{1}<\eta_{2}<1,
$$

we want

$$
0 \leq \Gamma<1 \text {, that is } 0 \leq \alpha_{1} \eta_{1}+\alpha_{2} \eta_{2}<1 \text {. }
$$


For $\mathcal{K}(s) \geq 0$, as in Lemma 5.1 we obtain the conditions

$$
\alpha_{1} \eta_{1}+\alpha_{2} \eta_{2} \geq 0, \quad \alpha_{1}+\alpha_{2} \geq 0 \text {. }
$$

The total requirement is

$$
0 \leq \alpha_{1} \eta_{1}+\alpha_{2} \eta_{2}<1, \quad \alpha_{1}+\alpha_{2} \geq 0
$$

We do not state the obvious theorems that follow from an application of Theorem 4.2. The authors of [8] study a problem with more complicated BCs which in their concluding remarks are noted to include (1.4) under some conditions, but they do not have theorems comparable with ours. As far as we know, this is the first time the conditions for these 4-point problems (and similarly $m$ point problems) have been determined.

Three other BCs are equivalent to the three we have just considered via the change of variable $t \mapsto 1-t$. These are:

$$
\begin{array}{ll}
u(0)=\alpha[u], & u(1)=0 ; \\
u(0)=\alpha[u], & u^{\prime}(1)=0 ; \\
u^{\prime}(0)+\alpha[u]=0, & u(1)=0 .
\end{array}
$$

Results for these BCs follow immediately from our theorems. Many other BCs can also be treated by our methods, we have merely indicated some of these to which our general method applies.

\section{More general equations}

We shall show how our general approach can be applied to equations of the form

$$
P u(t):=-\left(p(t) u^{\prime}(t)\right)^{\prime}+r(t) u(t)=g(t) f(t, u(t)), t \in(0,1)
$$

where $p(t)>0, r(t) \geq 0$ subject to the same types of BCs as studied above. The equation

$$
a(t) u^{\prime \prime}(t)+b(t) u^{\prime}(t)+c(t) u(t)+g(t) f(t, u(t))=0
$$

can be transformed into the form in (6.1) by multiplying by

$$
\frac{1}{a(t)} \exp \left(\int_{0}^{t} \frac{b(s)}{a(s)} d s\right) \text {. }
$$

Since typically we suppose $g \in L^{1}, a(t)$ could possibly be 0 at some points.

We briefly indicate how the method applies to the BCs $u(0)=0, u(1)=\alpha[u]$ when $\alpha[u]=\int_{0}^{1} u(s) d A(s)$ for a signed Stieltjes measure.

We suppose that the BVP

$$
P u=y, \quad u(0)=0, u(1)=0
$$


has a unique solution given by $u(t)=\int_{0}^{1} G(t, s) y(s) d s$ where $G$ is the Green's function which can be expressed in the form

$$
G(t, s)=\frac{1}{p W} \begin{cases}\phi_{1}(s) \phi_{2}(t) & \text { if } s \leq t, \\ \phi_{1}(t) \phi_{2}(s) & \text { if } s>t\end{cases}
$$

where $\phi_{1}$ is the unique positive solution of $P \phi_{1}=0, \phi_{1}(0)=0, \phi_{1}(1)=1$ and $\phi_{2}$ is the unique positive solution of $P \phi_{2}=0, \phi_{2}(0)=1, \phi_{2}(1)=0, W\left(\phi_{1}, \phi_{2}\right)$ is the Wronskian, and $p W$ is a (positive) constant by Abel's formula. If we define an operator $G$ by $G u(t):=\int_{0}^{1} G(t, s) g(s) f(s, u(s)) d s$, then

$$
P(G u)(t)=g(t) f(t, u(t)), G u(0)=0, G u(1)=0 .
$$

We can then find solutions of the BVP

$$
P(u)(t)=g(t) f(t, u(t)), u(0)=0, u(1)=\alpha[u]
$$

as fixed points of

$$
T u(t)=\phi_{1}(t) \alpha[u]+G u(t) .
$$

By our general theory we need the following conditions to be satisfied:

$$
0 \leq \alpha\left[\phi_{1}\right]<1, \quad \mathcal{K}_{G}(s)=\int_{0}^{1} G(t, s) d A(t) \geq 0 .
$$

Since these are integral (or sum) conditions, this certainly allows some sign changes as previously. Ma and Ren [17] treated the $m$-point problem with positive coefficients and obtained results on the existence of one and two positive solutions using the methods of $[10,11]$. We therefore have several types of improvement on these results: we can allow coefficients that change sign, or have continuously distributed signed measures, or a combination of these, and we allow weaker conditions on the nonlinearity using 'eigenvalues', etc.

Similarly we can treat the other BCs listed above. We do not state the plethora of results that can now be proved by a simple application of Theorems 4.1, 4.2, and their extensions, even though our results for measures are completely new.

The disadvantage of studying the general form of equation (6.1) is that one cannot check the conditions unless $\phi_{1}$ and $G(t, s)$ are known explicitly. This is why we have concentrated on the problems where we can verify the hypotheses.

\section{References}

[1] H. AMANN, Fixed point equations and nonlinear eigenvalue problems in ordered Banach spaces, SIAM. Rev., 18 (1976), 620-709.

[2] D. GUO and V. LAKSHMIKANTHAM, Nonlinear Problems in Abstract Cones, Academic Press, 1988. 
[3] CH. P. GUPTA, S. K. NTOUYAS and P. CH. TSAMATOS, Existence results for m-point boundary value problems. Differential Equations Dynam. Systems 2 (1994), 289-298.

[4] CHAitAN P. GUPTA and SERGEI I. TROFIMCHUK, Solvability of a multi-point boundary value problem and related a priori estimates, Canad. Appl. Math. Quart. 6 (1998), 45-?60.

[5] G. INFANTE and J. R. L. WEBB, Positive solutions of some nonlocal boundary value problems, Abstr. Appl. Anal., (2003), 1047-1060.

[6] G. INFANTE and J. R. L. WEBB, Nonlinear nonlocal boundary value problems and perturbed Hammerstein integral equations, Proc. Edinb. Math. Soc., 49 (2006), 637-656.

[7] G. L. KARAKOSTAS and P. CH. TSAMATOS, Existence of multiple positive solutions for a nonlocal boundary value problem, Topol. Methods Nonlinear Anal. 19 (2002), 109-121.

[8] G. L. KARAKOSTAS and P. CH. TSAMATOS, Multiple positive solutions of some Fredholm integral equations arisen from nonlocal boundary-value problems, Electron. J. Differential Equations 2002, No. 30, 17 pp.

[9] M. A. KRASNOSEL'SKII and P. P. ZABREIKKO, Geometrical methods of nonlinear analysis, Springer-Verlag, Berlin, 1984.

[10] K. Q. LAN, Multiple positive solutions of semilinear differential equations with singularities, J. London Math. Soc, 63 (2001), 690-704.

[11] K. Q. LAN and J. R. L. WEBB, Positive solutions of semilinear differential equations with singularities, J. Differential Equations, 148 (1998), 407-421.

[12] XIAOYING LIU, Nontrivial solutions of singular nonlinear $m$-point boundary value problems, J. Math. Anal. Appl. 284 (2003), 576-590.

[13] XIUJUN LIU, JIQING QIU and YANPING GUO, Three positive solutions for second-order $m$-point boundary value problems, Applied Math. Comp. 156 (2004), 733-742.

[14] RUYUN MA, Positive solutions of a nonlinear $m$-point boundary value problem, Comput. Math. Appl. 42 (2001), 755-765.

[15] RUYUN MA, Positive solutions for second order functional differential equation, Dynam. Systems Appl. 10 (2001), 215-223.

[16] RUYUN MA and NELSON CASTANEDA, Existence of solutions of nonlinear m-point boundary value problems, J. Math. Anal. Appl., 256 (2001), $556-567$. 
[17] RUYUN MA and LISHUN REN, Positive Solutions for nonlinear $m$-point boundary value problems of Dirichlet Type via fixed-point index theory, Applied Mathematics Letters 16 (2003), 863-869.

[18] R. H. MARTIN, Nonlinear operators and differential equations in Banach spaces, Wiley, New York, (1976).

[19] J. R. L. WEBB, Remarks on positive solutions of three point boundary value problems, Dynamical systems and differential equations (Wilmington, NC, 2002), Discrete Contin. Dynam. Systems 2003, Added Volume, 905-915.

[20] J. R. L. WEBB, Optimal constants in a nonlocal boundary value problem, Nonlinear Anal., 63 (2005), 672-685.

[21] J. R. L. WEBB and K. Q. LAN, Eigenvalue criteria for existence of multiple positive solutions of nonlinear boundary value problems of local and nonlocal type, Topol. Methods Nonlinear Anal., 27 (2006), 91-115.

[22] GUOWEI ZHANG and JINGXIAN SUN, Positive solutions of $m$-point boundary value problems, J. Math. Anal. Appl. 291 (2004), 406-418.

Received 2 December 2004, accepted 31 May 2005;

published Online First 10 January 2008

To access this journal online: http://www.birkhauser.ch 